\title{
The Mass Growth and Stellar Ages of Galaxies: Observations versus Simulations
}

\author{
Allison R. Hill ${ }^{1}$ (1) Adam Muzzin $^{2}$ (1) Marijn Franx $^{1}$ (1), and Danilo Marchesini ${ }^{3}$ (1) \\ ${ }^{1}$ Leiden Observatory, Leiden University, P.O. Box 9513, 2300 RA, Leiden, The Netherlands; hill@strw.leidenuniv.nl \\ ${ }^{2}$ Department of Physics and Astronomy, York University, 4700 Keele Street, Toronto, ON MJ3 1P3, Canada \\ ${ }^{3}$ Physics and Astronomy Department, Tufts University, 574 Boston Avenue, Medford, MA 02155, USA \\ Received 2017 August 14; revised 2017 October 19; accepted 2017 October 20; published 2017 November 3
}

\begin{abstract}
Using observed stellar mass functions out to $z=5$, we measure the main progenitor stellar mass growth of descendant galaxies with masses of $\log M_{*} / M_{\odot}=11.5,11.0,10.5,10.0$ at $z \sim 0.1$ using an evolving cumulative number density selection. From these mass growth histories, we are able to measure the time at which half the total stellar mass of the descendant galaxy was assembled, $t_{a}$, which in order of decreasing mass corresponds to redshifts of $z_{a}=1.28,0.92,0.60$, and 0.51 . We compare this to the median light-weighted stellar age $t_{*}$ $\left(z_{*}=2.08,1.49,0.82\right.$ and 0.37$)$ of a sample of low-redshift SDSS galaxies (from the literature) and find the timescales are consistent with more massive galaxies forming a higher fraction of their stars ex situ compared to lower-mass descendants. We find that both $t_{*}$ and $t_{a}$ strongly correlate with mass, which is in contrast to what is found in the EAGLE hydrodynamical simulation that shows a flat relationship between $t_{a}$ and $M_{*}$. However, the semi-analytic model (SAM) of Henriques et al. is consistent with the observations in both $t_{a}$ and $t_{*}$ with $M_{*}$, showing that the most recent SAMs are better able to decouple the evolution of the baryons from the dark matter in lower-mass galaxies.
\end{abstract}

Key words: galaxies: evolution - galaxies: formation

\section{Introduction}

Inferring the assembly history of present-day galaxies is challenging. It requires accurately linking progenitor to descendant, a process that is obfuscated by the fact that we only ever observe a galaxy one snapshot in time. However, by using mass-complete censuses of galaxies at different redshifts and observing how populations of galaxies move through various parameter spaces (i.e., SFR, sSFR, central surface mass density, central stellar velocity dispersion, number density, etc.), one can begin to connect descendant galaxies to their likely progenitor population.

By tracing galaxy evolution using a variety of the aforementioned parameters, observational studies are united in the finding that massive galaxies assemble most of their stellar mass before low-mass galaxies, indicative of baryonic "down-sizing" (e.g., Pérez-González et al. 2008; Marchesini et al. 2009; Behroozi et al. 2013; Muzzin et al. 2013; González Delgado et al. 2017). This is consistent with analyses of the stellar populations of local galaxies, which find that more massive galaxies are host to older stellar populations (e.g., Kauffmann et al. 2003; Gallazzi et al. 2005; Thomas et al. 2010).

In contrast to observations, semi-analytic models (SAMs) and hydrodynamical simulations do not share the same consistency. Although both hydrodynamical simulations and SAMs reproduce the positive correlation of stellar age with stellar mass, they differ in their predictions for when that mass assembled. Recent SAMs predict massive galaxies forming earlier than their low-mass counterparts (e.g., Henriques et al. 2015), in contrast to recent hydrodynamical simulations that show either a flat relationship between assembly time (the time at which $50 \%$ of the mass was assembled) and stellar mass ( $\mathrm{Qu}$ et al. 2017), or a weak positive correlation (Sparre et al. 2015).

Although these models are inconsistent with each other on trends of stellar mass with assembly, they do all predict that a higher fraction of the stars in massive galaxies were formed ex situ (e.g., Rodriguez-Gomez et al. 2016; Mundy et al. 2017; Qu et al. 2017). This picture is consistent with observations that indicate mergers are an important avenue of mass growth in massive galaxies since $z \sim 1$ (e.g., Newman et al. 2012; Hill et al. 2017). However, the role of mergers in the mass growth of lower-mass galaxies remains uncertain.

In this Letter, we endeavor to draw a direct observational comparison between the assembly time and the mass-weighted stellar age of galaxies and demonstrate more concretely the relationship between galaxy stellar mass and the fraction of ex situ stars. We also compare these timescales to the EAGLE simulation as well as the recent SAM of Henriques et al. (2015, hereafter H2015).

Unless otherwise specified, all ages and assembly times are for galaxies corresponding to a reference redshift of $z=0.1$, with all ages reported in lookback times. We assume a $\Lambda$-CDM cosmology $\left(H_{0}=70 \mathrm{~km} \mathrm{~s}^{-1} \mathrm{Mpc}^{-1}, \Omega_{M}=0.3\right.$, and $\left.\Omega_{\Lambda}=0.7\right)$.

\section{Analysis \\ 2.1. Measuring the Assembly Times}

To estimate the assembly time $\left(t_{a}\right)$ for a galaxy, we must first determine a mass assembly history. The first challenge to analyzing the mass evolution of present-day galaxies is properly identifying their progenitors. There are several methods to do this, e.g., by inferring the mass growth from the evolution of the SFR-mass relation (e.g., Patel et al. 2013a), selection via central surface mass density (e.g., van Dokkum et al. 2014), selection via fixed central velocity dispersion (e.g., Bezanson et al. 2012), and the evolution of the stellar mass function (e.g., Pérez-González et al. 2008; Marchesini et al. 2009; Muzzin et al. 2013; among others). The simplest and most appropriate method to derive the progenitor masses of galaxies is through cumulative number density selection. This method begins with the simple assumption that cumulative density would remain constant if there were no 

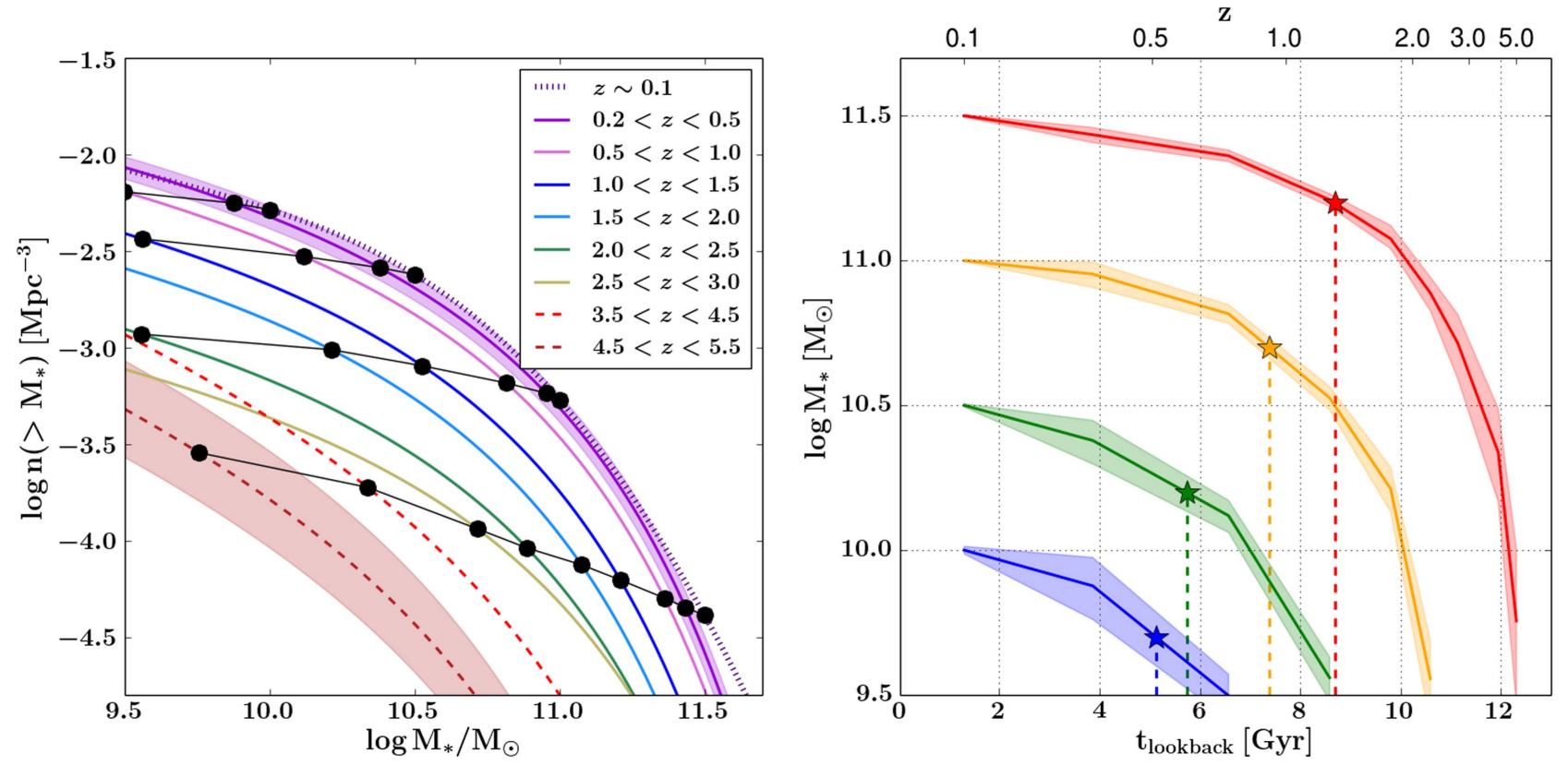

Figure 1. Left: the cumulative number density as a function of stellar mass at different $z$. Solid, dashed, and dotted lines indicate the mass functions of Muzzin et al. (2013), Grazian et al. (2015), and Bernardi et al. (2017), respectively, with color indicating the redshift. Uncertainties in the mass functions take into consideration the uncertainties in the photo-z, SFH, and cosmic variance. For clarity, only the uncertainties for the highest- and lowest- $z$ are shown (as the uncertainties monotonically increase with $z$ ). Black circles indicate the cumulative number density selection of Behroozi et al. (2013) for four different descendant masses at $z \sim 0.1$ $\left(\log M_{*} / M_{\odot}=11.5,11.0,10.5,10.0\right)$. Right: the corresponding mass evolution of the descendants considered in the left panel. Shaded regions indicate the uncertainty in the progenitor mass from the uncertainties in the mass functions. We trace the progenitors of four different descendant masses at $z \sim 0.1$. Also plotted are the assembly times ( $t_{\text {assembly }}$; colored stars), which are the times at which half the final descendant mass is assembled.

mergers or scatter in assembly; the evolution in the cumulative density due to these effects can be predicted robustly from models (Behroozi et al. 2013). These predictions have been tested and verified against more detailed simulations that accurately recover the median mass evolution (e.g., Torrey et al. 2015; Clauwens et al. 2016; Wellons \& Torrey 2017). This method is the only method that can give a fair estimate from the evolution of the mass function alone, i.e., it does not need any detailed modeling to the full $f\left(M_{*}\right.$, SFR, merger rate) distribution of galaxies.

In Figure 1, we show the number density and progenitor mass evolution for four different descendant masses of $\log M_{*} / M_{\odot}=11.5,11.0,10.5,10.0$ at $z \sim 0.1$. As in Hill et al. (2017), we utilize the mass functions of Muzzin et al. (2013) and Grazian et al. (2015) (with the addition of Bernardi et al. 2017 to extend to $z \sim 0.1$ ) to translate the number densities from Behroozi et al. (2013) into galaxy stellar masses as a function of redshift (left panel of Figure 1). The regular evolution of the implied progenitor mass as a function of redshift in the right panel highlights the quality of the input mass functions. Also indicated in the right panel of Figure 1 are the assembly times, $t_{a}$, the points at which half the final stellar masses were assembled. For our progenitor selection, this corresponds to assembly redshifts (in order of decreasing stellar mass) of $z_{a}=1.28,0.92,0.60,0.51$. In this plot, we see a clear trend toward baryonic cosmic "down-sizing," with the most massive galaxies assembling half their stellar mass earlier.

\subsection{Measuring the Stellar Ages}

To compare $t_{a}$ to the present-day age of the stellar populations in those galaxies, $t_{*}$, we take the light-weighted ages from Gallazzi et al. $\left(2005 ; t_{*, \mathrm{LW}}\right)$ which were measured from a subsample of 44,254 SDSS galaxy spectra. This subsample was chosen such that the median $\mathrm{S} / \mathrm{N}$ per pixel was greater than 20, in order to accurately, and simultaneously model both the age and metal sensitive spectral indices such as $\mathrm{H} \beta, \mathrm{H} \delta_{A}, \mathrm{H} \gamma_{A}, D 4000$, and $\left[\mathrm{Mg}_{2} \mathrm{Fe}\right]$. They also were careful to exclude galaxies at redshifts that deviated substantially from the Hubble flow, resulting in a redshift range of $0.005<z<0.22$, with a median redshift of $z=0.13$. Extensive and careful modeling, using a library of 150,000 Monte Carlo realizations that cover a wide parameter space of plausible star formation histories, were used to accurately determine both age and metallicity as well as to quantify the magnitude of the errors on these derived quantities. A full description of their methods can be found in Gallazzi et al. (2005).

For a galaxy with a given $M_{*}$, we take the median $t_{*, \mathrm{LW}}$ (see Table 2 in Gallazzi et al. 2005). As $t_{*, \mathrm{LW}}$ is a median value, the formal errors are small (fractions of a percentage point), so we do not include those errors. However, as the SDSS fibers impose an aperture, there is potential for errors resulting from age gradients, especially in the larger galaxies. A recent analysis of age gradients in SDSS galaxies by Goddard et al. (2017) found gradients at a level of $\sim 0.1 \mathrm{dex} / R_{e}$ from the center to $1.5 R_{e}$. This translates to an aperture correction of approximately $10 \%$, which we use as a conservative error estimate in the median $t_{*, \mathrm{LW}}$.

Light-weighted ages are biased toward younger stellar populations, as young stars dominate the optical emission where many age sensitive indices are measured (see Kauffmann et al. 2003). A more representative $t_{*}$ metric is the mass-weighted age, $t_{*, \mathrm{MW}}$. Since the SFH is not known for these galaxies, we generate stellar-mass-dependent corrections to $t_{*, \mathrm{MW}}$ using the differences 


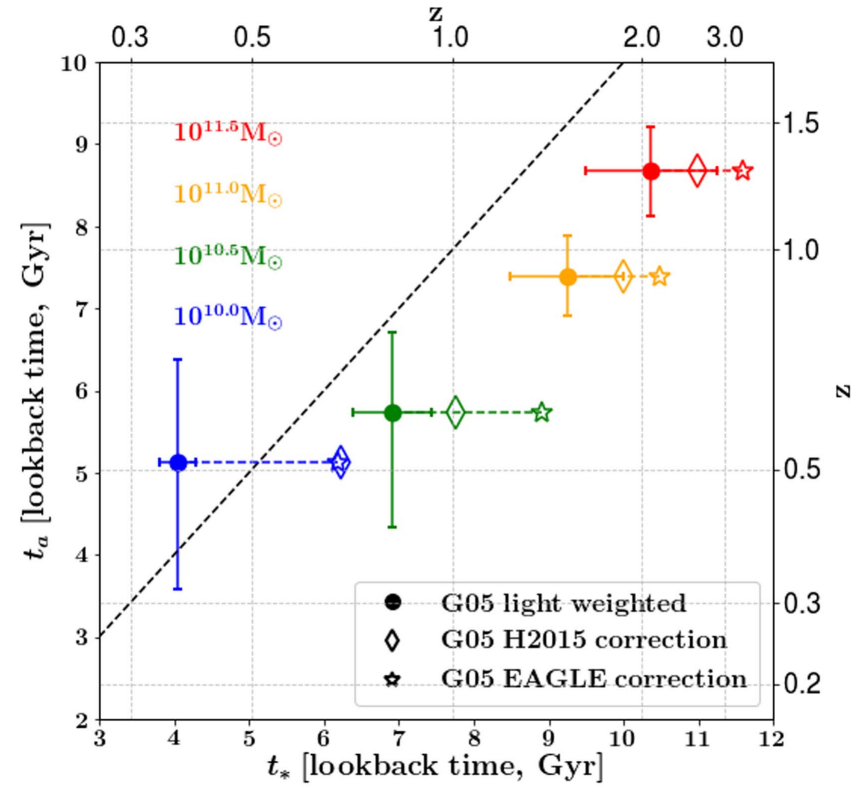

Figure 2. Assembly age, $t_{a}$, plotted as a function of the stellar age, $t_{*} \cdot t_{a}$ is defined as the age at which half the stellar mass was assembled, as determined from the stellar-mass evolution tracks in the right panel of Figure 1, with the errors estimated from uncertainties from the stellar mass functions. The stellar ages are the median light-weighted ages (filled circles) taken from Gallazzi et al. (2005), with the errors representing the expected uncertainty resulting from age gradients (see the text for details). We have also estimated a massweighted age correction to the light-weighted ages using corrections measured from both EAGLE and H2015 (details can be found in the main text). We see a positive correlation between $t_{*}, t_{a}$, and mass, with the most massive galaxies assembling first.

between the mass-weighted ages and r-band weighted ages from both H2015 (available in their catalog) and EAGLE (J. Trayford 2017, private communication) and apply it in the following way:

$$
t_{*, \mathrm{MW}, \mathrm{G} 05}=t_{*, \mathrm{LW}, \mathrm{G} 05}+\left(t_{*, \mathrm{MW}, \mathrm{sims}}-t_{*, \mathrm{LW}, \mathrm{sims}}\right) .
$$

Figure 2 shows $t_{a}, t_{*, \mathrm{MW}, \mathrm{G} 05}$ for both H2015 and EAGLE, and $t_{* \mathrm{LW}, \mathrm{G} 05}$ for all of our descendant galaxy masses. We see a range of assembly times, from $\sim 5 \mathrm{Gyr}$ at the low-mass end, to almost $9 \mathrm{Gyr}$ for our highest-mass bin. The span is larger in $t_{*}$, where we see a range of $\sim 5-11$ Gyr. We see all values are consistent with $t_{a}<t_{*, \mathrm{MW}}$, which confirms our results are physical. We observe $t_{*}-t_{a}$ increasing with stellar mass, which suggests a higher fraction of the stars in massive galaxies are formed ex situ than at lower masses. When comparing $t_{*}-t_{a}$ to the ex situ fractions of the H2015 SAMs, they imply an ex situ fraction of between $3 \%$ and $33 \%$ for $\log M_{*} \geqslant 10.5$ and between $1 \%$ and $33 \%$ for $\log M_{*}=10.0$. This finding is consistent with other observational studies (e.g., most recently, Rodríguez-Puebla et al. 2017, who use sub-halo abundance matching to find a $M_{*} \sim 5 \times 10^{11} M_{\odot}$ galaxy has $\sim 36 \%$ of their mass formed ex situ compared to only $\sim 2.4 \%$ for Milky Way mass galaxies). This trend is also seen in simulations (see Section 1 and references therein).

\subsection{Comparison to Simulations}

In Figure 3, we compare our assembly times, and the stellar ages of Gallazzi et al. (2005) to the median values of those found in the EAGLE simulation (Schaye et al. 2015), and the SAM of H2015 as a function of stellar mass. In this figure, we record the median r-band weighted stellar age of a narrow stellar mass range $\left(\Delta \log M_{*} / M_{\odot}=0.05\right)$ of galaxies from the largest EAGLE simulation (Ref-L100N1504) at $z=0.1$ and the millennium simulation (Henriques2015a..MRscPlanck1). We also trace the mass evolution of the most massive progenitors of theses galaxies to estimate an assembly redshift.

Figure 3 shows that the observations display a positive correlation between $t_{*}, t_{a}$, and stellar mass (as implied by the mass functions), assuming the relationships are of the form

$$
t_{*} \propto \alpha \log M_{*}, \quad t_{a} \propto \beta \log M_{*},
$$

where $\alpha$ and $\beta$ are the best-fit linear slopes for $t_{*}$ and $t_{a}$, respectively. The H2015 model also reproduces the positive trend between stellar mass, $t_{*}$, and $t_{a}$, albeit with slightly flatter slopes. For observations, we find $\alpha_{\text {obs }}=4.25 \pm 0.55$ and $\beta_{\text {obs }}=2.46 \pm 0.30$, which are both steeper than the those implied for $\mathrm{H} 2015\left(\alpha_{\mathrm{H} 2015}=3.42 \pm 0.56, \beta_{\mathrm{H} 2015}=1.38 \pm\right.$ 0.34 ) although they agree to within $2 \sigma$. This suggests the SAMs are doing a good job at reproducing the formation of stars and their assembly for the stellar mass ranges considered in this study, with a slight bias toward earlier formation.

The EAGLE simulation similarly reproduces the relationship between $t_{*}$ and $M_{*}$, although the value for $\alpha$ is even flatter than that of H2015 $\left(\alpha_{\text {EAGLE }}=2.19 \pm 0.39\right)$. For the assembly time, EAGLE does not reproduce the trend at all, and instead has a $\beta$ consistent with $0\left(\beta_{\mathrm{EAGLE}}=-0.17 \pm 0.40\right)$. This is also seen in Qu et al. (2017), who performed a more robust analysis of the EAGLE simulation galaxy assembly, and whose median assembly times also indicate a flat relationship with $M_{*}$.

At high masses $\left(M_{*}=10^{11.5} M_{\odot}\right)$, compared to observations, massive EAGLE galaxies assemble their mass too quickly. There are also issues at the lowest mass where the discrepancy of stellar ages and assembly times in EAGLE at $\log M_{*} / M_{\odot}=10.0$ is significant (and also present in $\mathrm{H} 2015$, although not as discrepant in the SAM) and likely related to simulations overproducing low-mass galaxies at higher redshift (see Weinmann et al. 2012; Henriques et al. 2013; Lacey et al. 2016).

\section{Discussion and Conclusions}

From Figures 1 and 2 , we see a clear trend between $t_{a}$ and $t_{*}$ with stellar mass. More massive galaxies formed earlier, and at $\log M_{*} / M_{\odot} \geqslant 10.5$, they also have stellar ages that are older than their respective assembly times, suggesting that a larger fraction of their stars formed ex situ compared to lower-mass galaxies. This picture implies that mergers are a more important component of stellar mass growth in massive galaxies, which is consistent with what is seen in previous studies (e.g., Naab et al. 2009; Hopkins et al. 2010; van Dokkum et al. 2010; Trujillo et al. 2011; Newman et al. 2012; Hilz et al. 2013; McLure et al. 2013; Vulcani et al. 2016; Hill et al. 2017; Mundy et al. 2017). In contrast, with $t_{a} \approx t_{*}$ for galaxies at $\log M_{*} / M_{\odot}<10.5$, almost all the stars can be attributed to in situ formation.

Although the $t_{a}$ was not calculated explicitly, both Patel et al. (2013b) and van Dokkum et al. (2013) used fixed cumulative number density arguments to calculate the stellar mass evolution as a function of redshift, from which a $z_{a}$ can be inferred. Using their fits for $M_{*}(z)$, for a $10^{11.2} M_{\odot}$ galaxy, Patel et al. (2013b) found an assembly redshift of 1.97 . Using our prescription, for the same galaxy mass, we would find $z_{a}=1.42$. van Dokkum et al. (2013), for a $10^{10.7} M_{\odot}$ galaxy, 

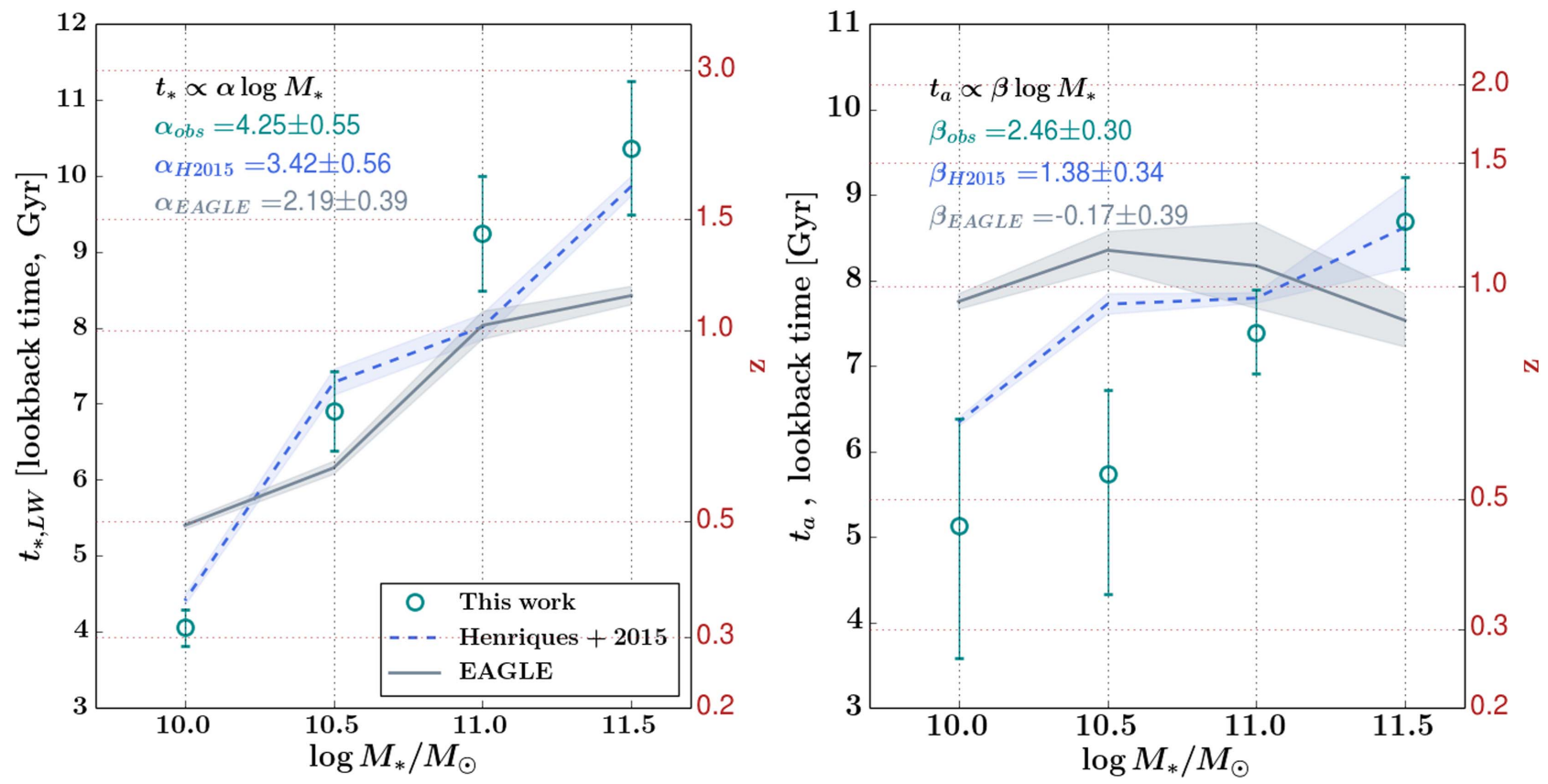

Figure 3. Stellar age ( $t_{*}$; left panel) and assembly age $\left(t_{a}\right.$; right panel) plotted as function of their final stellar masses. The turquoise circles are the values determined from observations (as in Figure 2), solid gray lines are median values from the EAGLE simulation Ref-L100N1504, and dashed blue lines are from H2015. with the shaded regions around these lines representing the error implied from bootstrapping the samples. We see the same positive correlation between $t_{a}$ and $t_{*}$ with stellar mass as implied in Figure 2. The simulations also show this positive correlation with $t_{*}$ and $M_{*}$, with H2015 better matching (to within $2 \sigma$ ) the steep dependence than EAGLE, which has a flatter relationship than the observations imply. In the right panel, we see the same flat relationship between $M_{*}$ and $t_{a}$ in EAGLE as found in Qu et al. (2017), which does not match the observations, and is in fact consistent with a slope of 0 . The $t_{a}$ measured from H2015 are in good agreement with the observations (to within $2 \sigma$ ).

find $z_{a}=1.35$ (and for which we would find $z_{a}=0.91$ ). Both studies find earlier assembly redshifts than we do. About half this redshift discrepancy is due different selection criteria (i.e., the use of a fixed cumulative number density instead of an evolving cumulative number density, where the former predicts higher mass progenitors; see Hill et al. 2017), with the remainder due to the use of different mass functions.

A comparison of our results to recent hydrodynamical simulations (Schaye et al. 2015) and SAMs (H2015) show good agreement in the relationship of $t_{*}$ with stellar mass in all but the lowest-mass bin (with the exception of the highest-mass bin in the EAGLE simulation). This is especially impressive in EAGLE considering the models were not calibrated to reproduce stellar ages. The disagreement in $t_{*}$ in the lowestmass bin suggests either simulations are still forming stars too early, or, conversely, the stellar ages in lower-mass galaxies are underestimated. Using deep $(\mathrm{S} / \mathrm{N}(\AA)>50)$ spectroscopy of a handful of local group galaxies, Sánchez-Blázquez et al. (2011) found that nearby barred-spiral galaxies were dominated by stars with ages on the order of $\sim 10$ Gyr. Using deep, colormagnitude diagrams of local dwarfs, Hidalgo et al. (2013) also found that the majority of stars in local dwarfs are between 9 and 10 Gyr old. This is in apparent contradiction to the median ages found by Gallazzi et al. (2005). It is possible that the smaller local samples are not representative of the population as a whole. Conversely, the reverse could also be true and that the low-mass end of the galaxies from Gallazzi et al. (2005) are also not representative. Alternatively, one way to resolve the discrepancy is to assume that there is a positive relation between stellar mass and age, which has a turnover at dwarfgalaxy stellar masses (although this seems unlikely). A more robust survey of low-mass, and hence low-surface brightness, galaxies would be needed to address these issues.
If we assume that the mass-weighted stellar ages inferred from Gallazzi et al. (2005) are correct, then the disagreement between observations and EAGLE of $t_{*}$ also folds into the assembly times, where we see more significant disagreement between EAGLE and our estimates (although with large scatter). EAGLE does not reproduce the positive correlation between $t_{a}$ and stellar mass, but instead predicts a flat relationship that might be related to the fact that EAGLE does not reproduce the GSMF (Furlong et al. 2015).

Considering the SAMs of a decade ago (e.g., De Lucia et al. 2006), there has been massive improvement, with the assembly times calculated from the most recent SAM (H2015) agreeing remarkably well with the observations (to within $2 \sigma$ ), with a slight bias to early assembly times. Although there have been great improvements in recent modeling and simulation work in regard to reproducing the GSMF, these results suggest that there are potential systematic offsets that need to be addressed and that the evolution of the baryonic component of low-mass galaxies has not been sufficiently decoupled from their host dark-matter halos. Observationally, there is an underexplored parameter space in regard to low-mass galaxies, which are crucially needed to inform the simulations.

\section{Summary}

In this Letter, we have measured the assembly time and stellar ages from observations for four different mass descendant galaxies $\left(\log M_{*} / M_{\odot}=11.5,11.0,10.5,10.0\right)$ at $z \sim 0.1$ and find:

1. The assembly times and stellar ages decrease with decreasing stellar mass, consistent with cosmic "downsizing." 
2. The difference between $t_{a}$ and $t_{*}$ increases weakly with increasing stellar mass, suggesting that massive galaxies form a larger fraction of their stars ex situ compared to lower-mass galaxies.

3. $t_{a}$ and $t_{*}$ both increase with stellar mass, ranging from $\sim 5$ to $11 \mathrm{Gyr}$ in mass-weighted stellar age and $\sim 5-9 \mathrm{Gyr}$ in assembly times. The SAM model of H2015 reproduces these trends to within $2 \sigma$, albeit with slightly flatter relationships. EAGLE reproduces the positive correlation with $t_{*}$, but not with $t_{a}$ where EAGLE predicts no mass dependence on assembly times.

4. The assembly times and stellar ages from the most recent SAM from the Millennium simulations (Henriques et al. 2015) are in good agreement with the observations, with a slight bias to earlier formation and assembly.

We would like to thank Bruno Henriques and James Trayford for deriving values from the H2015 and EAGLE catalogs, respectively. We are also grateful to Pieter van Dokkum and the anonymous referee, whose comments greatly improved the presentation of this work. D.M. acknowledges the National Science Foundation under grant No. 1513473. This research has made use of NASA's Astrophysics Data System.

\section{ORCID iDs}

Allison R. Hill (ํ) https://orcid.org/0000-0002-0696-5458 Adam Muzzin (1) https://orcid.org/0000-0002-9330-9108 Marijn Franx (ํ) https://orcid.org/0000-0002-8871-3026 Danilo Marchesini (i) https://orcid.org/0000-0001-9002-3502

\section{References}

Behroozi, P. S., Marchesini, D., Wechsler, R. H., et al. 2013, ApJL, 777, L10 Bernardi, M., Meert, A., Sheth, R. K., et al. 2017, MNRAS, 467, 2217 Bezanson, R., van Dokkum, P., \& Franx, M. 2012, ApJ, 760, 62 Clauwens, B., Franx, M., \& Schaye, J. 2016, MNRAS, 463, L1

De Lucia, G., Springel, V., White, S. D. M., Croton, D., \& Kauffmann, G. 2006, MNRAS, 366, 499

Furlong, M., Bower, R. G., Theuns, T., et al. 2015, MNRAS, 450, 4486
Gallazzi, A., Charlot, S., Brinchmann, J., White, S. D. M., \& Tremonti, C. A. 2005, MNRAS, 362, 41

Goddard, D., Thomas, D., Maraston, C., et al. 2017, MNRAS, 466, 4731

González Delgado, R. M., Pérez, E., Cid Fernandes, R., et al. 2017, A\&A, in press (arXiv:1706.06119)

Grazian, A., Fontana, A., Santini, P., et al. 2015, A\&A, 575, A96

Henriques, B. M. B., White, S. D. M., Thomas, P. A., et al. 2013, MNRAS, 431, 3373

Henriques, B. M. B., White, S. D. M., Thomas, P. A., et al. 2015, MNRAS, 451, 2663

Hidalgo, S. L., Monelli, M., Aparicio, A., et al. 2013, ApJ, 778, 103

Hill, A. R., Muzzin, A., Franx, M., et al. 2017, ApJ, 837, 147

Hilz, M., Naab, T., \& Ostriker, J. P. 2013, MNRAS, 429, 2924

Hopkins, P. F., Bundy, K., Hernquist, L., Wuyts, S., \& Cox, T. J. 2010, MNRAS, 401, 1099

Kauffmann, G., Heckman, T. M., White, S. D. M., et al. 2003, MNRAS, 341,33

Lacey, C. G., Baugh, C. M., Frenk, C. S., et al. 2016, MNRAS, 462, 3854

Marchesini, D., van Dokkum, P. G., Förster Schreiber, N. M., et al. 2009, ApJ, 701, 1765

McLure, R. J., Pearce, H. J., Dunlop, J. S., et al. 2013, MNRAS, 428, 1088

Mundy, C. J., Conselice, C. J., Duncan, K. J., et al. 2017, MNRAS, 470, 3507

Muzzin, A., Marchesini, D., Stefanon, M., et al. 2013, ApJ, 777, 18

Naab, T., Johansson, P. H., \& Ostriker, J. P. 2009, ApJL, 699, L178

Newman, A. B., Ellis, R. S., Bundy, K., \& Treu, T. 2012, ApJ, 746, 162

Patel, S. G., Fumagalli, M., Franx, M., et al. 2013a, ApJ, 778, 115

Patel, S. G., van Dokkum, P. G., Franx, M., et al. 2013b, ApJ, 766, 15

Pérez-González, P. G., Rieke, G. H., Villar, V., et al. 2008, ApJ, 675, 234

Qu, Y., Helly, J. C., Bower, R. G., et al. 2017, MNRAS, 464, 1659

Rodriguez-Gomez, V., Pillepich, A., Sales, L. V., et al. 2016, MNRAS, 458,2371

Rodríguez-Puebla, A., Primack, J. R., Avila-Reese, V., \& Faber, S. M. 2017, MNRAS, 470, 651

Sánchez-Blázquez, P., Ocvirk, P., Gibson, B. K., Pérez, I., \& Peletier, R. F. 2011, MNRAS, 415, 709

Schaye, J., Crain, R. A., Bower, R. G., et al. 2015, MNRAS, 446, 521

Sparre, M., Hayward, C. C., Springel, V., et al. 2015, MNRAS, 447, 3548

Thomas, D., Maraston, C., Schawinski, K., Sarzi, M., \& Silk, J. 2010, MNRAS, 404, 1775

Torrey, P., Wellons, S., Machado, F., et al. 2015, MNRAS, 454, 2770

Trujillo, I., Ferreras, I., \& de La Rosa, I. G. 2011, MNRAS, 415, 3903

van Dokkum, P. G., Bezanson, R., van der Wel, A., et al. 2014, ApJ, 791, 45

van Dokkum, P. G., Leja, J., Nelson, E. J., et al. 2013, ApJL, 771, L35

van Dokkum, P. G., Whitaker, K. E., Brammer, G., et al. 2010, ApJ, 709, 1018

Vulcani, B., Marchesini, D., De Lucia, G., et al. 2016, ApJ, 816, 86

Weinmann, S. M., Pasquali, A., Oppenheimer, B. D., et al. 2012, MNRAS, 426, 2797

Wellons, S., \& Torrey, P. 2017, MNRAS, 467, 3887 